This file is a pre-print and may contain errors or omissions not presented in the final published version.

\title{
Why do bilingual code-switch when emotional? Insights from immigrant parent-child interactions
}

\author{
Aya Williams, University of California, Berkeley \\ Mahesh Srinivasan, University of California, Berkeley \\ Chang Liu, The Pennsylvania State University \\ Pearl Lee, University of California, Berkeley \\ Qing Zhou, University of California, Berkeley
}

In press at Emotion

\begin{abstract}
Author Note
This research was supported by a grant from the Child Development Young Scholars Program and Hellman Family Fund awarded to Qing Zhou. Work on this manuscript has been supported by the National Science Foundation Graduate Research Fellowship awarded to Aya Williams and a grant from the Eunice Kennedy Shriver Institute of Child Health \& Human Development to Qing Zhou and Yuuko Uchikoshi. The authors wish to thank all parents and children who participated in and contributed to the study. Special thanks to Stephen Chen and Iris Mauss for suggestions on the content of this document. Correspondence should be addressed to Qing Zhou at the Department of Psychology at the University of California, Berkeley, 2121 Berkeley Way, CA 94704. Email: qingzhou@berkeley.edu
\end{abstract}




\begin{abstract}
Previous research has found that bilingual speakers' first (L1) and second languages (L2) are differentially associated with their emotional experiences. Moreover, bilinguals appear to code-switch (alternate between two or more languages in a single conversation) during emotional episodes. However, prior evidence has been limited to clinical case studies and self-report studies, leaving open the specificity of the link between code-switching (CS) and emotion, and its underlying mechanisms. The present study examined the dynamic associations between CS and facial emotion behavior in a sample of 68 Chinese-American parents and children during a dyadic emotion-inducing puzzle box task. Specifically, bilingual parents' language use (L1 Chinese or L2 English), CS behavior (L1 $\rightarrow$ L2 or L2 $\rightarrow$ L1 switches), and facial emotion behavior (positive and negative valence) were coded at each 5-second interval. Multilevel modeling was used to analyze whether facial emotion behavior predicted later CS, and vice versa. We found that negative facial emotion predicted higher subsequent CS in both L1 $\rightarrow$ L2 and L2 $\rightarrow$ L1 directions, with stronger associations for the L2 $\rightarrow \mathrm{L} 1$ direction. On the other hand, positive facial emotion was associated with lower contemporaneous L2 $\rightarrow$ L1 CS. CS did not predict later facial emotion behavior, suggesting language switching may not have an immediate effect on emotion. The present findings are consistent with the idea that emotional arousal, especially negative arousal, reduces cognitive control and may trigger spontaneous CS. Together, these findings provide insight into why bilingual speakers switch languages during emotional episodes, and hold implications for clinical interventions serving bilingual individuals and families.
\end{abstract}

Keywords: bilingual, code-switching, emotion, immigrant, parent-child 


\section{Introduction}

"We usually argue in English/Spanish mish mash. When either of us are boiling, it is each [in] her/his own L1. When I am arguing, yet feeling clever and witty and relaxed, I will often use my L2 to deliver some really poisonous barbs.” (L1 English, L2 Spanish; Pavlenko, 2005, p. 135; emphasis added)

Bilingual speakers often observe that their first (L1) and second languages (L2) are differentially associated with their emotional experiences, and that this can guide their choice of which language to use (Pavlenko, 2005). These observations have raised fundamental questions about the link between language and emotion, and more specifically, the use of code-switching (CS; i.e., alternation between two or more languages within a single conversation, Myers-Scotton, 1993) as a potential tool for managing one's emotions. Evidence of a CS-emotion link, however, has been limited to clinical case studies of bilingual patients (Marcos \& Alpert, 1976; Rozensky \& Gomez, 1983; Javier, 1989; Aragno \& Schlachet, 1996; Mohavedi, 1996; Foster, 1996; Santiago-Rivera \& Altarriba, 2002) and self-report studies (Pavlenko, 2004, 2005, 2012; Dewaele, 2008, 2010, 2015; Dewaele \& Nakano, 2012), leaving open the robustness and specificity of this relation, as well as the mechanisms that might give rise to it.

In the present study, we examined the dynamic associations between CS and facial behavior by observing bilingual parents and children during a dyadic emotion-inducing puzzle box task (Eisenberg et al., 2001). In a sample of Chinese American parent-child dyads, we measured the frequency and directionality of parents' CS, as well as the valence and intensity of their facial emotion behavior. Using multilevel models, we tested the moment-to-moment associations between the parent's CS and facial emotion behavior. The present findings can 
inform theories on why bilingual speakers code-switch during emotional episodes. The results will have broader implications for interventions serving bilingual individuals and their families. Previous Research on the Link between CS and Emotion in Bilingual Speakers

Broadly defined, CS refers to the bilingual speaker's practice of alternating between two languages in the context of a single conversation (Myers-Scotton, 1993). Types of CS range from insertion of single words to the alternation of languages for larger segments of discourse (Muysken, 2000). Though CS has been historically viewed with stigma (e.g., as a sign of mental confusion), recent theoretical and empirical work substantiates a view of CS as a form of linguistic competence that requires a high degree of grammatical and pragmatic ability in both languages (e.g., Meisel, 1994). In particular, CS is highly patterned and structurally governed (Myer-Scotton, 1993). It tends to occur at predictable points that do not violate the syntactic rules of either language (Poplack, 1980). Moreover, bilingual speakers have been found to use CS to achieve various communicative effects (Gardner-Chloros, 2009), index social roles and identities (Gumperz, 1982), and manage ongoing conversation (Gafaranga, 2009).

Researchers have long asked the question: why do bilingual speakers code-switch? A link between CS and emotional experiences is plausible under at least three theoretical perspectives. First, from a cognitive control perspective, CS arises from the competition for the most appropriate language to convey meaning at a particular moment in time. Given that multiple languages are active in the bilingual speaker's mind (Costa, Miozzo, \& Caramazza, 1999), a control process is required in selecting the appropriate language. According to the adaptive control hypothesis, distinct control processes are engaged in different interactional contexts: single language context (one language is used in one environment and the other in a second distinct environment), dual language context (both languages are used but typically with 
different speakers), or CS context (speakers routinely interleave their languages in the course of a single utterance) (Green \& Abutalebi, 2013; Green \& Wei, 2014). Broadly, competitive control is engaged in single and dual language contexts, whereas cooperative control is engaged in CS context (Green \& Wei, 2016). Under competitive control, only a single target language is permitted entry into the speech plan. Under cooperative control - the context studied in the present study - more than one language can be part of the speech plan. Thus, in the latter, a control mechanism is required to select (or suppress) words and constructions in the appropriate language for speech output.

Heightened emotion may interfere with cooperative control processes involved in language selection, thereby increasing the frequency of CS. Previous studies have provided evidence of a lower capacity for cognitive control in emotional contexts than in those without (Tottenham, Hare \& Casey, 2011). In particular, studies have found that processing of negative emotional stimuli reduced top-down control by diverting cognitive resources (Cohen et al., 2016). Thus, a bilingual speaker's cognitive control may be temporarily disrupted during negative emotional episodes. Because both L1 and L2 items enter the speech plan under cooperative control, reduced control permits free and opportunistic use of L1 and L2 items irrespective of language membership, which may increase CS during speech output. Consistent with this proposal, one lesion study found that a patient with disrupted cognitive control spontaneously code-switched even when instructed not to do so (Fabbro, Skrap, \& Aglioti, 2000). CS during emotional episodes may therefore result from the influence of heightened emotion on cooperative control processes.

Second, from the emotion regulation perspective, bilingual speakers may code-switch to regulate the intensity of their emotions. Specifically, bilingual speakers may switch to their 
native L1 to express more intense emotions (i.e., up-regulate) and switch to their second language L2 to express less intense emotions (i.e., down-regulate) (Pavlenko, 2005, 2014). Researchers have postulated that L1 and L2 become differentially associated with emotion through the process of learning and habitually using each language in distinct emotional contexts. For example, L1 is typically learned and used in more emotional contexts such as the home, and L2 is typically learned and used in less emotional contexts such as the foreign language classroom (Caldwell-Harris, 2014). This can result in a less automatic processing of L2, triggering weaker emotional responses (Thoma \& Baum, 2018). Empirical studies have shown that bilingual speakers experienced increased emotion when speaking in L1 than in L2, as indicated by their facial emotion behavior, pupil dilation, vocal tone, and autonomic responses (see Pavlenko, 2012 for a review). In qualitative self-reports, bilinguals have described L1 use during emotional episodes as "natural," "sincere," and "intimate" in contrast to L2 use as "fake," "wrong," and "artificial," highlighting differences in the perceived fidelity to the emotional experience. Overall, this relation between language and emotion appears to be causal, such that bilingual speakers experience increased emotions in response to L1 stimuli and decreased emotions in response to L2 stimuli (Caldwell-Harris, 2014).

Observations from clinical case studies further suggest that it is possible for bilingual speakers to strategically use CS to change their emotional trajectory, or regulate. CS among bilingual patients have been documented across Spanish/English (Rozensky \& Gomez, 1983; Marcos \& Alpert, 1976; Javier, 1989; Aragno \& Schlachet, 1996; Foster, 1996; Santiago-Rivera \& Altarriba, 2002), German/English (Marcos \& Alpert, 1976; Aragno \& Schlachet, 1996), and Persian/English (Mohavedi, 1996) languages. Emotion disruptions during psychotherapy, such as crying and display of anger accompanied switching into L1 (Rozensky \& Gomez, 1983). More 
psychotic symptoms were also found in L1 (Javier, 1989). Among patients with trauma history, use of L1 prompted the recall of trauma, such as childhood abuse ("in German I am a scared dirty child, in English I am a nervous refined woman” Marcos \& Alpert, 1976). In contrast, patients described greater emotional control in L2 ('It's just that in English, I can express myself so much better. I know exactly what I want to say,” Foster, 1996). A more recent study (Santiago-Rivera, et al., 2009) in fact found that Spanish/English patients used L2 English to reduce emotional intensity when feeling frightened.

Finally, from a cultural frame switching perspective, bilingual speakers may code-switch to adhere to culturally expected patterns of emotion (Ervin-Tripp, 1964; Ross, Xun, \& Wilson, 2002; Mesquita, Boiger, \& De Leersnyder, 2017; Wang, Shao, \& Li, 2010). Cultural factors shape ideal affect, or affective states that people strive toward (Tsai, 2007). For instance, members of individualistic cultures may value high arousal positive states (e.g., excitement) more than those from collectivistic cultures. Language then may serve as a vehicle for transmitting culturally distinct affective goals. Consistent with this, American parents were found to use more positive emotion terms defined by high arousal positive states when interacting with their children, compared to Chinese parents (Camras et al., 2006). Relatedly, Chinese American parents self-reported CS from Chinese into English to say "I love you" to their children (Pavlenko, 2005, p. 136; Ross, Xun, \& Wilson, 2002). Emotion words are not universal across cultural groups and translation non-equivalence (e.g., "cariño" in Spanish does not have a direct translation equivalent in English) may trigger CS to allow bilingual speakers to express emotion in a culturally congruent manner (Wierzbicka, 2005). Beyond the cultural differences in emotion words or concepts (Lindquist, Gendron, \& Satpute, 2018), bilingual speakers may choose one language as more appropriate than another language for conveying 
their emotion consistent with a particular cultural frame (Panayiotou, 2004; also labeled as emotional frame switching; De Leersnyder \& Mesquita, 2017).

The three perspectives described above are theoretically distinct, despite some conceptual overlap. The cognitive control perspective primarily focuses on how emotion influences language (i.e., emotional change as a trigger for CS), whereas the emotion regulation and cultural frame switching perspectives focus on how language influences emotion (i.e., emotional change as a function of CS). From the cognitive control perspective, emotion is thought to reduce cognitive control, which permits the use of words and constructions from both languages, thereby increasing CS. From the emotion regulation and cultural frame switching perspectives, language shapes the emotional experience with availability of words or its associations with previous experiences in emotional contexts. Even in the cases where emotion may trigger a regulatory $\mathrm{CS}$, the theoretical focus of the emotion regulation and cultural frame switching accounts is on the downstream effects of language on emotion. Between the emotion and culture perspectives, the main distinction lies in the extent to which cultural linguistic factors influence emotion. In general, the emotion regulation perspective suggests a more 'deep' effect of language on emotion, such that L1/L2 differences should be observed at the physiological level (e.g., skin conductance response). The cultural frame switching perspective, on the other hand, suggests a more 'shallow' effect at the level of self-reported experiences and affective goals. This difference may be due to the emphasis on the context of language acquisition from the emotion regulation perspective (i.e., the order of acquisition is thought to heavily influence the emotionality of L1/L2, in addition to L1/L2 use in emotional contexts). Consequently, L1 is viewed as the most emotional language from the emotion regulation perspective, whereas L2 may become as emotional as L1 through acculturation by the cultural frame switching account. 
These theoretical perspectives make different predictions about the types of codeswitches that may occur during emotional episodes. Muysken (2000) identified three types of CS: insertion (lexical items from one language are inserted into the grammatical structure of another language), alternation (grammatical structures switch between languages), and congruent lexicalization (lexical items from both languages are used in shared grammatical structure, also labeled dense CS). From the cognitive control perspective, heightened emotion may reduce control such that both L1 and L2 items are produced freely and opportunistically (i.e., open control), resulting in dense CS (Green \& Wei, 2014). From the emotion regulation and cultural frame switching perspectives, alternation between L1 and L2 or insertion of specific emotion words or phrases (e.g., "I love you" in English) into another language may cause changes in the speaker's subsequent facial emotion behavior (Pavlenko, 2005; Mesquita et al., 2017).

\section{Methodological Considerations for Understanding the CS-Emotion Link}

In sum, previous research has found evidence of a possible link between CS and emotional experiences. However, as we discuss below, existing studies have also left open the robustness and specificity of the CS-emotion relation due to methodological limitations. For example, the widely used Bilingualism and Emotion Questionnaire (BEQ; Dewaele \& Pavlenko, 2001-2003) consisted of single-item questions about subjective experiences (e.g., "Do you switch between languages when talking about certain matters neutral, personal, or emotional?"). An important limitation of using self-report measures to assess emotional experience is that individuals are not always reliable reporters of their emotional states (Levenson, 1999). Internal states are often difficult to describe in words, and large individual differences exist in one's emotional awareness and granularity (Lindquist \& Barrett, 2008). Emotion researchers have indeed found modest coherence between self-reported emotional experience, behavior, and 
physiological responses (Mauss, et al., 2005). Thus, it remains an empirical question whether the CS-emotion associations documented in self-reported subjective experiences could be extended to observed behavioral measures, such as in-vivo CS and facial emotion behaviors.

Moreover, although case studies and self-report studies have provided qualitatively rich accounts of emotion-related CS, systematic comparisons of emotional valence (positive or negative) and CS directionality ( $\mathrm{L} 1 \rightarrow \mathrm{L} 2$ or $\mathrm{L} 2 \rightarrow \mathrm{L} 1$ switches) have yet to be examined quantitatively. According to self-reports, some bilingual parents reported CS to express negative emotions (e.g., disciplining, scolding, conflict), whereas others have reported CS to express positive emotions (e.g., endearment, affection) (Pavlenko, 2004). Similarly, clinicians have observed CS in both $\mathrm{L} 1 \rightarrow \mathrm{L} 2$ and $\mathrm{L} 2 \rightarrow \mathrm{L} 1$ directions among bilingual patients to express or manage intense emotional experiences during psychotherapy (Krapf, 1955; Mohavedi, 1996; Javier, 1989). A potential interpretation is that bilingual speakers may code-switch to mark an affective stance; however, the specific explanations or mechanisms underlying emotion-related CS remain unclear. In sum, examining the specific associations between valence and CS directionality could potentially explain these mixed findings, building on existing theories.

Furthermore, prior studies have not adequately taken into account the potential covariates that might confound the CS-emotion link. Researchers have often grouped together bilingual speakers across a range of individual and contextual factors, including language proficiency, acquisition history, topic of conversation, degree of monolingual to bilingual mode activation in the immediate speech context, and acculturation (Gardner-Chloros, 2009). These variables can influence the likelihood of CS as well as facial emotion behavior. In the present study, we used an ethnically homogeneous sample of Chinese American immigrant families with similar language acquisition and proficiency profiles. All parent-child dyads completed the same puzzle 
box task, which constrained the topic of conversation, along with instructions provided in both L1 and L2. In our analyses, we identified five covariates when testing the CS-emotion links. First, we examined acculturation (i.e., length of stay in the US) which has previously been associated with both CS use (Ng \& He, 2004) and facial display rules (Matsumoto, 1993). Second, we controlled for bilingual proficiency (i.e., parent's and children's Chinese and English proficiency), which has been found to moderate CS (Ribot \& Hoff, 2014) and influence facial emotion behavior (Pavlenko, 2012). Finally, children's positive and negative facial emotion behaviors at each 5-second interval were also taken into account, as they may influence parent's facial behavior (Lindsey, Cremeens, Colwell, \& Caldera, 2009).

\section{CS-Emotion Link within the Bilingual Immigrant Family}

Immigrant families offer an ideal context for studying the CS-emotion link, because bilingual CS is a prevalent and naturally occurring phenomenon within this population (National Academies of Sciences, Engineering, and Medicine, 2017). A common language profile of immigrant families is one in which parents are more dominant in their heritage language than in the host language (Buriel, Love, \& De Ment, 2006). In contrast, children typically learn the parent's heritage language in the home context, and become more dominant in the host language as they enter school (Fillmore, 1991). This acculturation gap often results in frequent CS in parent-child conversation, while parents and children experience various daily emotions.

The present study sampled parent-child dyads in Chinese American immigrant families, which provided an opportunity to test two competing theories on the CS-emotion relation. Specifically, parents in our sample were sequential bilingual speakers who had acquired L1 Chinese in the home context during childhood followed by L2 English in school or upon arriving in the US at a later age. The parents had spent on average 16 years in the US $(S D=7.98)$ and 
were sufficiently exposed to both Chinese and American cultural norms and goals related to emotion. This language history of the parents is consistent with the histories of participants who have shown differential affective processing in L1 (more emotional) and L2 (less emotional) in previous studies (Harris, Gleason, \& Ayçiçegi, 2006). Thus, based on the emotion regulation theory, we hypothesized that parents may code-switch from L2 (English) into L1 (Chinese) to express more intense emotion, and from L1 to L2 to express less intense emotion, as measured by facial emotion behavior (Pavlenko, 2005). An alternative prediction stems from the fact that Chinese and American cultural groups have distinct display rules (Soto, Levenson, \& Ebling, 2005; Chen, Zhou, Main, \& Lee, 2015), whereby Chinese culture places more emphasis on suppression or inhibition of emotion (Chao, 1995), whereas American culture places higher value on open expression. Specifically, Chinese culture places greater value on low arousal positive affect, whereas American culture places greater value on high arousal positive affect (Tsai, 2007). Given these culturally distinct norms and goals about emotion, the cultural frame switching theory would alternatively predict that parents may code-switch from L2 to L1 (Eastern framework) to exhibit less intense emotion, and from L1 to L2 (Western framework) to exhibit more intense emotion, especially for positive states.

\section{The Present Study}

The present study examined the dynamic associations between parent's CS and facial behavior in a sample of Chinese American parent-child dyads during a 5-min emotion-inducing puzzle box task. Parents and children's intensity of observed facial emotion behavior (positive and negative valence) were coded for each 5-second epoch. Parent's language choice (Chinese or English), and CS frequency and directionality (L1 $\rightarrow \mathrm{L} 2$ or $\mathrm{L} 2 \rightarrow \mathrm{L} 1$ switch) were also coded during each epoch. Using multilevel modeling, we tested the moment-to-moment associations 
between prior facial emotion behavior and subsequent CS frequency, and between prior CS frequency and subsequent facial emotion behavior.

The methods allowed us to test several competing hypotheses about the CS-emotion link generated from the theoretical perspectives reviewed above. First, based on the cognitive control theory, we expected more intense facial emotion behavior would be associated with increased subsequent CS frequency (Abutalebi \& Green, 2007), and that this relationship would be stronger for negative states (Cohen et al., 2016). Additionally, based on the emotion regulation (Pavlenko, 2005) and cultural frame switching theories (Mesquita, Boiger, \& De Leersnyder, 2017), we expected CS at an earlier time point would predict changes in later facial emotion behavior. Specifically, according to the emotion regulation theory, L2 $\rightarrow$ L1 CS frequency should increase intensity of facial emotion behavior, while L1 $\rightarrow$ L2 CS should decrease intensity of facial emotion behavior. In contrast, according to the cultural frame switching theory, L2 $\rightarrow \mathrm{L} 1$ CS frequency into an Eastern cultural frame should decrease intensity of facial emotion behavior, whereas L1 $\rightarrow$ L2 CS frequency into a Western cultural frame should increase intensity of facial emotion behavior, especially for positive states (Tsai, 2007).

\section{Method}

\section{Participants}

The present study was part of a larger, longitudinal study of psychological, social, and academic adjustment of Chinese-American children from immigrant families in the San Francisco Bay Area (Chen et al., 2014). A variety of recruitment strategies were employed, including partnerships with schools and community organizations with large Asian populations, and wide distribution of study fliers at recruitment fairs in Chinese American communities to recruit a socioeconomically diverse sample. Inclusion criteria were as follows: (a) the child was 
enrolled in first- or second-grade at the time of initial screening, (b) the child lived with at least one biological parent, (c) both biological parents identified as ethnically Chinese, (d) the child was either a first- or second-generation immigrant, and (e) both parent and child were able to understand and speak English and Chinese (Mandarin or Cantonese). The study was approved by the Institutional Review Board.

The full study sample consisted of 258 first (24\%) and second generation (76\%) Chinese American children ( $48 \%$ girls, $\left.M_{\text {age }}=7.4, S D=.71\right)$, their parents, and their teachers. Of these parents, 74\% were born in China, 9\% born in Hong Kong, 3\% in Taiwan, and 14\% born in other parts of the world. The parents had lived in the US for an average of 12 years (range $=1$ to 38 years, $S D=7.63)$. Parental education was on average 13 years (range $=5$ to 20 years, $S D=2.50)$. Annual per capita income was $\$ 11,607$ (range $=\$ 625$ to $\$ 50,000, S D=\$ 8,309)$, calculated by dividing the estimated total family income for the past year by the number of adults and children living in the household. All participants were Chinese/English bilingual speakers. With respect to language proficiency, the parents were on average more proficient in Chinese $(M=4.2, S D=.78$; $1=$ Extremely poor, $3=$ Average, $5=$ Very good $)$ than in English $(M=2.7, S D=.95)$. On the other hand, the children were slightly more proficient in English $(M=3.5, S D=.74)$ than in Chinese $(M=2.8, S D=.85)$.

To observe CS behavior, the present subsample was selected based on the parents' balanced use of L1 Chinese and L2 English during the puzzle box task (Tao, et al., 2013). Parent's language use was coded using a 5-point Likert scale that ranged from -2 to 2 (-2 = parents spoke Chinese only, $-1=$ parents spoke more Chinese than English, $0=$ parents used equal amounts of English and Chinese, 1 = parents spoke more English than Chinese, and $2=$ parents spoke English only). From the larger study sample, the 44 dyads who scored between -1 
and 1 inclusive were selected into a subsample of relatively balanced bilingual speakers. Of these, 8 video recordings were unable to be coded due to parent or child faces not appearing in the video frame for more than $50 \%$ of the study task, and 2 videos were missing. Thus, 34 parentchild dyads were included in the present sample. Comparison of the selected and unselected samples on key demographic variables (i.e., parent and child age, gender, income, education, number of years living in the US) and language variables (i.e., parent and child language proficiency, child age of exposure to English and Chinese) indicated no group differences, except the child's Chinese proficiency in the selected sample was lower $(p=.01)$.

The present subsample consisted of a total of 68 parents ( $88 \%$ mothers) and children (56\% girls). Of these parents, $61 \%$ were born in China, $9 \%$ born in Hong Kong, $5 \%$ born in Taiwan, and 25\% born in other countries outside of the US. The parents had lived in the US for an average of 16 years (range $=3$ to 34 years, $S D=7.98$ ). Parental education was on average 14 years (range 10 to 20 years, $S D=2.52$ ). The mean per capita income for the sample was $\$ 13,355$ (range $=\$ 1,000$ to $\$ 33,333, S D=\$ 8,029)$. The participating children were between 6 and 9 years of age $\left(M_{\text {age }}=7.3, S D=.81\right)$. Of these children, $15 \%$ were born outside of the US (i.e., $1^{\text {st }}$ generation), and $85 \%$ were born in the US (i.e., $2^{\text {nd }}$ generation). With respect to language proficiency, the parents were on average more proficient in Chinese $(M=4.1, S D=.95 ; 1=$ Extremely poor, $3=$ Average, $5=$ Very good $)$ than in English $(M=2.9, S D=.79)$. On the other hand, the children were slightly more proficient in English $(M=3.6, S D=.76)$ than in Chinese $(M=2.5, S D=.76)$.

We calculated the post-hoc power for the current analysis by using the observed effect size for key study parameters (Bolger \& Laurenceau, 2013). Specifically, we used Mplus to conduct Monte Carlo power simulation (Muthén \& Muthén, 2002). For the parameters that were 
significant, the calculated post-hoc power varied from 0.7 to 1.0 , indicating that the original study design was adequately powered to test some parameters, but may not be adequately powered to test some other parameters, based on the convention of .80 power or greater.

\section{Procedures and Measures}

Procedure. Each dyad completed a 2.5-hour laboratory assessment in the full study, which included a parent interview and questionnaires, child interview and behavioral tasks, and parent-child dyadic interaction tasks. All questionnaires and interviews were administered in the parent's and child's preferred language (English, Mandarin, or Cantonese) by bilingual ChineseAmerican research assistants. All written materials (i.e., informed consent forms and parent questionnaires) were provided in English, simplified Chinese, and traditional Chinese.

Family demographics and migration history (parent-report). The Family

Demographics and Migration History Questionnaire was adapted from a similar measure used in a study of Mexican American immigrant families (Roosa et al., 2008). The questionnaire included questions on maternal and paternal education, employment, family annual income, parent's and child's country of birth, reason for immigration, and length of stay in the US.

Parent's and child's Chinese and English proficiency (parent-report). The Cultural and Social Acculturation Scale (CSAS; Chen \& Lee, 1996) is a bidimensional scale that assesses the individual's contact and engagement with both heritage and host cultures. The CSAS is available in Chinese and English, and has shown satisfactory internal reliabilities in a previous study of Chinese American mothers and their children (Garrett-Peters \& Fox, 2007). The present study used the language domain of the scale, which measures speaking, understanding, reading, and writing abilities in English and Chinese. The parents rated their own proficiency as well as their child's proficiency on a Likert scale ranging from one to five points ( 1 = Extremely poor, 3 
$=$ Average, $5=$ Very good). The alpha reliabilities for the full sample were .95 and .91 for English and Chinese respectively.

Parent's and child's facial emotion behavior (observed). Parent's and child's facial emotion behaviors were measured using a parent-child puzzle box task developed by Eisenberg and colleagues (2001) to observe a range of parent's positive and negative facial emotion behavior while interacting with their children. In the 5-minute puzzle box task, the child was shown a wooden box that contained a puzzle with alphabet-shaped pieces inside. The box was constructed with clear Plexiglas on one side (so that the child's hand movements could be observed) and black cloth with sleeves on the other side through which the child could insert his or her arms. The parent was instructed to sit on the side of the clear Plexiglas and help the child complete the puzzle verbally. Both parent and child were instructed to complete the puzzle without the child looking at the puzzle pieces and told that the child would receive a prize if he or she completed the puzzle within five minutes. Two visible video cameras were placed in the assessment room to record the parent's and child's facial emotion behaviors.

Parent's and child's facial emotion behaviors were coded from video recordings of the task using a coding scheme developed by Eisenberg and colleagues (Eisenberg, et al., 2001). To supplement Eisenberg's coding scheme with visual physical cues of facial behaviors, we also used anger and enthusiasm codes of the Specific Affect Coding System (SPAFF; Coan \& Gottman, 2007). The original 30-second coding scheme was reduced to 5-second epochs to capture the moment-to-moment changes in facial behavior as well as language. For each 5second epoch during the 5-minute task, parent's and child's facial emotion behaviors was coded for positive and negative emotions on a Likert scale from 0 to 3 points $(0=$ neutral, $3=$ intense $)$. Global displays of positive emotion included smiling, laughter, alertness, and interest. Global 
displays of negative emotion included frowning, pressed lips, tightened jaw, and lowered eyebrows. To establish inter-rater reliability, three coders were trained to ensure consistent applications of code definitions. Coding was completed by two independent raters, who were trained on 10 videos to ensure consistent application of code definitions and rated 44 video recordings for another study. One main coder and one reliability coder, both bilingual and bicultural, rated all videos. When these two coders disagreed, the video segments were reviewed, discussed, and resolved with the third coder. The inter-rater reliabilities, as calculated by interclass correlations for positive and negative emotions for the present sample, were both .97 . Given the high inter-rater reliability, codes from one rater were used for analyses. All coding was carried out on muted videos to remove the influence of language.

Parent's and child's language choice and CS (observed). Parent's and child's language choice and CS was coded in the original languages from the video recordings of the parent-child puzzle box task using ELAN (Lausberg \& Sloetjes, 2009). For each 5-second epoch during the 5-minute task, a trained bilingual coder assessed whether the parent and child spoke in English, Chinese (Cantonese or Mandarin), or both. In addition, the bilingual research assistant coded every instance of CS, defined as an alternation between two or more languages within a conversational discourse (Myers-Scotton, 1993). CS was operationalized to include both intrasentential switches (containing at least one morphological or lexical element from two languages within the same sentence or clause), and inter-sentential switches (whereby a switch between two languages occurs outside of the sentence or clause level; Genesee, Nicoladis, \& Paradis, 1995). For example, “Yes, the next row, this is (係, 下一橫, 依個係; haih, hah yāthòhng, yigo haih) twenty, nineteen” would count as a single switch, whereas “Turn, no, not like that, turn, (唔係咁, 轉; ’̀h haih gam, jyún), rotate” would count as two switches. A change of language following a 
pause (i.e., 5-seconds of silence) was not considered as a switch. Turn-taking between interlocutors was also not counted as an inter-sentential switch (e.g., child speaks English and parent responds in Chinese). CS frequency was calculated as the sum of inter- and intrasentential switches during each 5-second epoch. Each code-switch was additionally coded for directionality as L2 English to L1 Chinese CS, or L1 Chinese to L2 English.

\section{Plan of Data Analysis}

Based on the recommended cutoffs of 2 and 7 for skewness and kurtosis respectively (West, Finch, \& Curran, 1995), all main study variables met the criterion for normality except parent's positive and negative facial emotion, which were positively skewed. We examined between- and within-person associations between parent's CS and parent's positive (PE) or negative facial emotion behavior (NE) using multilevel models (Snijders \& Bosker, 1999), while accommodating dependencies of the repeated measurement of individuals. Specifically, to test the hypotheses that parent's PE or NE would predict changes in CS over time, we conducted 1epoch lagged multilevel models. Similarly, to test the hypothesis that parent's CS would predict parent's PE or NE, we used 1-epoch lagged generalized multilevel models to accommodate the non-normality of emotion codes. Due to the parent-directed nature of the puzzle box task (Eisenberg et al., 2001; Chen et al., 2014) and the rarity of child CS (3.2\% of total epochs in the present sample), analyses were only conducted on parent's CS and facial emotion behaviors. All analyses were conducted using the Linear and Nonlinear Mixed Effects Models package (nlme) (Pinheiro et al., 2017) and Linear Mixed-Effects Models using Eigen and SE package (lme4) (Bates, Maechler, Bolker, \& Walker, 2014) in R (R Core Team, 2013).

Data preparation. For the three main study variables, $6.0 \%$ of the data was missing ( $9.0 \%, 9.0 \%$, and $0.1 \%$ for PE, NE, and CS respectively). Participants provided data for a total of 
$4,526$ of the 4,818 possible epochs ( $94 \%$ response rate) and $97 \%$ of parents and children ( $n=66)$ completed the study task. Missing data on facial emotion codes were due to participant movement during the task and the puzzle box covering a part of the participant's face. Thus, missing data was treated as missing completely at random for data analysis.

To prepare the data, we centered parent's PE and NE to independently test between- and within-person associations with CS (Bolger, Davis, \& Rafaeli, 2003). We separated person $i$ 's mean level of PE or NE from person $i$ 's state level of PE $\left(\right.$ State PE $E_{i}$ ) or NE (State $\left.N E_{i}\right)$. For instance, person $i$ 's mean level of PE was calculated as the within-person mean of his or her PE across the entire task, while person $i$ 's state level of PE at epoch $t$ was calculated as the deviation of PE at epoch $t$ from the individual mean level of PE. Similarly, we centered parent's CS and separated person $i$ 's mean level of CS from person $i$ 's state level of CS (State $\left.C S_{i}\right)$ to independently test between- and within-person associations with PE or NE.

Analysis 1: Parent's facial emotion behavior predicting CS frequency. In the first level (Level 1) within-person analysis, we predicted parents' CS at epoch $t$ from their (a) CS at epoch $t-1$, (b) emotion (PE or NE) at epoch $t$, and (c) emotion at epoch $t-1$. In the second level between-person analysis (Level 2), we tested whether there was a significant association between parents' expected frequency of CS and their mean PE or NE. The models were specified by the following equations:

Level 1:

$$
\begin{aligned}
C S_{i t}=\beta_{0 i}+ & \beta_{1 i} C S_{i(t-1)}+\beta_{2 i}(\text { State PE }(\text { or State } N E))_{i t} \\
& +\beta_{3 i}(\text { State PE }(\text { or State } N E))_{i(t-1)}+\varepsilon_{i t}
\end{aligned}
$$

Level 2:

$$
\beta_{0 i}=\gamma_{00}+\gamma_{01}\left(\text { Mean PE }(\text { or Mean NE) })_{i}+u_{0 i}\right.
$$




$$
\begin{gathered}
\beta_{1 i}=\gamma_{10}+u_{1 i} \\
\beta_{2 i}=\gamma_{20} \\
\beta_{3 i}=\gamma_{30}
\end{gathered}
$$

where $C S_{i t}$ is the observed CS frequency at epoch $t$ for person $i, \beta_{0 i}$ is a person-specific intercept, $\beta_{1 i}$ is a person-specific first-order autoregressive coefficient for CS, $\beta_{2 i}$ is a personspecific contemporaneous association between state level emotion at epoch $t$ and CS at epoch $t$, $\beta_{3 i}$ is a person-specific lagged association between state level emotion at epoch $t-1$ and CS at epoch $t$, and residual error, $\varepsilon_{i t} \cdot \gamma_{00}$ is the expected level of CS for a parent whose mean level of emotion was $0, \gamma_{01}$ is the between-person association between the mean level emotion and CS, $\gamma_{10}$ is the average within-person association between CS at epoch $t-1$ and CS at epoch $t, \gamma_{20}$ is the within-person contemporaneous association between state level emotion and CS, $\gamma_{30}$ is the within-person lagged association between state level emotion at epoch $t-1$ and CS at epoch $t$, and $u_{0 i}$ and $u_{1 i}$ are individual-level residual deviations, which were not correlated with the residual error $\varepsilon_{i t}$. We also examined $u_{2 i}$ and $u_{3 i}$, which were not significant, and thus removed.

Analysis 2: Parent's CS frequency predicting facial emotion behavior. We further examined the opposite temporal relations of parent's CS predicting facial emotion behavior using generalized multilevel model with count outcomes. The Level 1 within-person model predicted parent's emotion at epoch $t$ from their (a) emotion (PE or NE) at epoch $t-1$, (b) CS at epoch $t$, and (c) CS at epoch $t-1$. The Level 2 between-person model tested whether there was a significant association between parent's expected level of PE or NE and their mean frequency of CS. The models were specified by the following equations:

Level 1:

$$
\log _{e} P E(\operatorname{or} N E)_{i t}=\beta_{4 i}+\beta_{5 i} P E(\text { or NE) })_{i(t-1)}+\beta_{6 i}(C S)_{i t}+\beta_{7 i}(C S)_{i(t-1)}
$$


Level 2:

$$
\begin{gathered}
\beta_{4 i}=\gamma_{40}+\gamma_{41}(\text { Mean CS })_{i}+u_{4 i} \\
\beta_{5 i}=\gamma_{50}+u_{5 i} \\
\beta_{6 i}=\gamma_{60} \\
\beta_{7 i}=\gamma_{70}
\end{gathered}
$$

where $P E(\text { or } N E)_{i t}$, is the observed emotion at epoch $t$ for person $i, \beta_{4 i}$ is a person-specific intercept, $\beta_{5 i}$ is a person-specific first-order autoregressive coefficient for emotion, $\beta_{6 i}$ is a person-specific contemporaneous association between state level CS and emotion at epoch $t$, and $\beta_{7 i}$ is a person-specific lagged association between state level CS at epoch $t-1$ and emotion at epoch $t . \gamma_{40}$ is the expected level of facial emotion behavior for a parent whose mean level of CS was $0, \gamma_{41}$ is the between-person association between the mean level of CS and emotion, $\gamma_{50}$ is the average within-person association between emotion at epoch $t-1$ and at epoch $t, \gamma_{60}$ is the within-person contemporaneous association between CS and state level emotion, $\gamma_{70}$ specifies the within-person lagged association between CS at epoch $t-1$ and emotion at epoch $t$, and $u_{4 i}$ and $u_{5 i}$ are individual-level residual deviations. We also examined $u_{6 i}$ and $u_{7 i}$, which were not significant, and thus removed from the final models.

\section{Results}

On average, across all subjects and all epochs, bilingual parents code-switched 1.48 times per epoch $(S D=1.55)$ and expressed higher intensity of positive emotion $\left(M_{P E}=.38, S D=.76\right)$ than negative emotion $\left(M_{N E}=.20, S D=.47\right)$. With respect to language choice, parents used Chinese in $22 \%$, English in $16 \%$, and code-switched in $62 \%$ of the total epochs. When using Chinese, parents exhibited similar intensity of positive $\left(M_{P E}=.28, S D=.69\right)$ and negative $\left(M_{N E}\right.$ $=.25, S D=.57)$ facial emotion behaviors. In contrast, when using English, parents exhibited 
higher intensity of positive $\left(M_{P E}=.49, S D=.83\right)$ than negative $\left(M_{N E}=.11, S D=.33\right)$ facial emotion behaviors. With respect to the direction of CS, parents switched from L1 to L2 and vice versa equally $\left(M_{L 1} \rightarrow L 2=.74, S D=.83 ; M_{L 2} \rightarrow L 1=.74, S D=.83\right)$. Concurrent zero-order correlations indicated that parent's CS frequency was negatively associated with intensity of PE expression $(r=-.06, p=.03)$ and marginally positively associated with intensity of NE expression $(r=.05, p=.08)$. Moreover, PE and NE were negatively associated $(r=-.17, p<.01)$.

CS, PE, and NE exhibited considerable variability at both between- and within-person levels. Intra-class correlations, representing the proportion of between-person variance, ranged from .07 to .20 for these variables. The results for PE predicting CS are shown in Table 1. We found significant first-order autoregressive effects of CS such that the CS at epoch $t-1$ predicted increases in the CS at the subsequent epoch $t\left(\gamma_{10}=.17, p<.01\right)$. The random effect for this within-person association was significant $\left(\sigma_{\mathrm{u} 1}=.07\right.$, with $\left.95 \% \mathrm{CI}=[.01, .30]\right)$, suggesting that the first-order autoregressive effects varied across individuals. In addition, CS was not associated with mean level PE at the between-person level $\left(\gamma_{01}=.01, p=.98\right)$, indicating that CS was not influenced by the mean level of PE across the task. But CS showed a marginally significant contemporaneous association with the state level of PE at the within-person level $\left(\gamma_{20}=-.10, p\right.$ $=.08$ ). That is, parents exhibited less frequent CS when they expressed higher intensity of PE. The strength of association was stronger for L2 English to L1 Chinese switches $(\gamma=-.06, p$ $=.08)$ than L1 Chinese to L2 English switches $(\gamma=-.05, p=.11)$.

The results for NE predicting CS are also shown in Table 1. Consistent with findings from the models on PE, we found significant first-order autoregressive effects of CS $\left(\gamma_{10}=.17, \mathrm{p}\right.$ $<.01)$. The random effect for this within-person association was also significant $\left(\sigma_{\mathrm{u} 1}=.06\right.$, with $95 \% \mathrm{CI}=[.01, .37])$, indicating individual differences in the autoregressive effects. Furthermore, 
CS was not contemporaneously associated with the mean level of NE at the between-person level $\left(\gamma_{01}=.58, \mathrm{p}=.38\right)$, nor was the state level of NE at the within-person level $\left(\gamma_{20}=.04, \mathrm{p}=.64\right)$. Importantly, the CS at epoch $t$ was predicted by parent's NE at epoch $t-1\left(\gamma_{30}=.20, p=.02\right)$, such that parents with higher NE predicted increased frequency of CS at the next time point. Subsequent analyses indicated that the effect of negative facial emotion on code-switches was stronger for L2 English to L1 Chinese switches $(\gamma=.11, p=.02)$ than L1 Chinese to L2 English switches $(\gamma=.08, p=.08)$.

The results for CS predicting PE and NE are shown in Table 2. We found significant first-order autoregressive effects of PE such that the PE at epoch $t-1$ predicted increases in the PE at the subsequent epoch $t\left(\gamma_{50}=.61, p<.01\right)$. The random effect for this within-person association was significant $\left(\sigma_{\mathrm{u} 5}=.26\right.$, with $\left.95 \% \mathrm{CI}=[.13, .42]\right)$, suggesting that the first-order autoregressive effects of PE varied across individuals. However, the mean level of CS and the state level of CS at epoch $t$ and $t-1$ were not significant predictors of PE at epoch $t(p \mathrm{~s}>.05)$. When examining CS predicting NE, we found significant first-order autoregressive effects of NE $\left(\gamma_{50}=.85, p<.01\right)$. However, the random effect for this within-person association was not significant $\left(\sigma_{\mathrm{u} 5}=.20\right.$, with $\left.95 \% \mathrm{CI}=[.00, .45]\right)$, suggesting that the first-order autoregressive effects of NE did not vary across individuals. Furthermore, similar to the results for CS predicting PE, the mean levels of CS and the state levels of CS at epoch $t$ and $t-1$ were not significant predictors of NE at epoch $t(p s>.05)$.

To identify covariates for the analyses, we first examined pairwise correlations between the theorized potential confounding variables, facial emotion behavior, and CS frequency. Among the variables examined, none were significantly correlated with both parent's facial emotion behavior and CS. Although parent's English proficiency $(r=.36, p<.05)$ and child's 
English proficiency $(r=.40, p<.05)$ were positively correlated with parent's positive facial emotion behavior, they were unrelated to CS. Parent's Chinese proficiency was marginally correlated with parent's CS $(r=-.33, p<.10)$. Child's positive facial emotion behavior was negatively correlated with parent's CS $(r=-.36, p<.05)$, but unrelated to parent's facial emotion behavior. To examine whether the analyses were influenced by the potential confounding factors, we conducted additional analyses by including parent's bilingual proficiency, child's English proficiency, and child's positive facial emotion behavior. For PE as the predictor and CS as the outcome, results remained the same and parent's Chinese proficiency $(\beta=-.40, t=-2.41, p$ $<.05)$ and child's PE $(\beta=-1.03, t=-.53, p<.05)$ were significantly associated with parent's CS. For NE as the predictor and CS as the outcome, results remained the same and parent's Chinese proficiency $(\beta=-.21, t=-2.30, p<.05)$ and child's $\mathrm{PE}(\beta=-.50, t=-2.04, p=.05)$ were significantly associated with parent's CS. For facial emotion behavior as the outcome, the models did not converge when including the covariates. The nonconvergence may be due to the limited sample size of the study.

While the majority of the parental utterances were brief task-specific instructions (i.e., yes, no, right, left, turn), qualitative analysis of the data indicated some patterns on language choice and the specific types of code-switches used. With respect to language choice, numbers were almost exclusively stated in English (“Yes, the next row, this is (對, 下一排, 這是; duì, xià yi pái, zhè shi) twenty, nineteen, the adjacent one (旁邊的那一個; pángbiān dè nà yigè).” Praises such as "good," "perfect" and "good job" were also almost exclusively stated in English. With respect to CS, repetition and clarification appeared to be associated with CS in both directions (“It's the closest, the one closest to you (離你最近的那一個; lí nǐ zuìjin dè nà yigè), yes"). Statements conveying positive ("There you go, yay! Very good! What number is this? (這 
個是幾號; zhège shì jǔ hào)") and negative emotions ("No, not that one, you already put that one already (不是那個, 你已經放了那一個; bùshì nàgè, nì y̌̌jīng fàngle nà yigè), oh my God”) were often associated with CS in both directions.

\section{Discussion}

To our knowledge, this is the first study that has used observational measures to empirically test the dynamic moment-to-moment associations between CS and facial emotion behavior in a naturalistic context. Overall, we found that parent's positive facial emotion was associated with their concurrent CS in a negative direction (i.e., parents code-switched less frequently when showing more positive facial emotion). Furthermore, parent's negative facial emotion was prospectively associated with CS in a positive direction (i.e., parents code-switched more frequently after expressing more negative facial emotion). On the other hand, CS did not predict any subsequent changes in facial emotion behavior.

Our results were most consistent with the cognitive control theory for why bilingual speakers code-switch during emotional experiences. According to the adaptive control hypothesis, cognitive control facilitates the selection of the appropriate target language among two activated languages (Abutalebi \& Green, 2007; Green \& Abutalebi, 2013). We found that the intensity of parent's negative, but not positive, facial emotion predicted the frequency of CS shortly after. That is, bilingual parents code-switched more after displaying frustration or anger during the puzzle box task, whereas parents code-switched less when they displayed joy or enthusiasm. These differential effects of valence are consistent with previous evidence indicating increased cognitive control performance during positive emotion states, and decreased cognitive control performance during negative emotion states (Cohen et al., 2016). Heightened negative emotion may temporarily reduce cognitive resources for control engaged during language 
selection. Reduced cognitive control, in turn, may freely permit entry of words and constructions from both languages into speech output, resulting in more frequent CS.

Green and Wei (2014) explain that dense CS is more likely to occur in language pairs from the same language family because of shared grammatical structures. With respect to the type of CS found in our qualitative analyses, dense CS may not have been observed due to the distinct grammatical structures of Chinese and English. Thus, heightened negative emotion may have served as a momentary change reducing cognitive control and increasing CS, but limited to insertions and alternations among Chinese/English bilinguals. With respect to the direction of CS, parents were more likely to switch into L1 after exhibiting negative facial emotion. One interpretation is that L1 processing is more automatic, whereas L2 processing is more effortful and deliberate (Keysar et al., 2012). Thus, under reduced cognitive control, the more automatic L1 may be more easily activated and produced. However, this finding should be interpreted with caution, since asymmetry of CS may not be a reliable indicator of bilingual language control, especially among bilinguals with relatively balanced proficiency (Bobb \& Wodniecka, 2013).

A related question that motivated our study is whether CS might have an adaptive effect on emotional communication. Cognitive control and emotion regulation are intertwined processes implicated in similar brain regions (Ochsner \& Gross, 2005). Spontaneous CS due to reduced cognitive control may be similar to speech disfluency, which is associated with the interaction of lower emotion regulation and negative emotional arousal (Walden et al., 2012). A pause in speech is an example of a speech disfluency that allows speakers time to plan speech and access lexical items, thereby serving an adaptive function (Clark \& Fox Tree, 2002). Heightened negative emotion may trigger CS, per the cognitive control theory. However, this CS could in principle serve adaptive functions, supporting the emotion regulation theory (e.g., 
negative emotion triggers switch into L1 to up-regulate anger for disciplining or switch into L2 for down-regulating anger to instruct more calmly) or the cultural frame switching theory (e.g., negative emotion triggers switch into English to be more emotionally expressive within the Western cultural frame). In our moment-to-moment analysis, we did not find evidence for CS predicting subsequent changes in facial emotion behavior. One explanation for this null finding may be due to the frequency of CS that we observed. Parents in our sample code-switched rapidly, as indicated by frequency that ranged between 0 to 9 switches per 5 -second interval ( $M$ $=1.48, S D=1.55)$. It is possible that changes in facial emotion behavior will only result from a code-switch that is maintained for a longer timespan (i.e., language choice).

Indeed, we found partial support for the cultural frame switching theory with respect to language choice. First, we found significant correlations between parent's $(r=.36, p<.05)$ and child's $(r=.40, p<.05)$ English use and parent's positive facial emotion. This is consistent with the idea that the use of English constitutes the American cultural frame in which high arousal positive states are valued more relative to Chinese cultural frame (Tsai, 2007). Second, in the qualitative portion of our study, we found that praise (e.g., "good job") was almost exclusively stated in English, again reflecting Western positive parenting norms. Similar to insertion of emotional phrases such as "I love you" reported in previous studies (Dewaele, 2008), explicit verbalization of praise has been found to be more common in culturally Western parenting styles (Chao, 1995). Although we did not find consistent patterns in our moment-to-moment relations between CS and facial emotion, the descriptive data on language choice and the parental communication of praise provide support for the cultural frame switching account.

The present study had several limitations. First, since we controlled for potentially confounding variables by selecting a relatively homogenous population of bilinguals, our 
findings may not generalize to all bilingual speakers. The present findings are most relevant for immigrant adults who are sequential bilingual speakers (i.e., who have learned one language in early childhood, and another language later on). Second, due to limited sample size, the study did not have adequate power to detect the effects of covariates. Future studies with larger sample sizes could examine which idiographic factors, such as cognitive control skills, might predict individual variations in emotion-related CS. Third, facial emotion behavior alone is not a bona fide measure of felt emotion (Fernandez-Dols \& Russell, 2017), given the moderate associations between emotional experience and behavior (Mauss et al., 2005). Still, the present study demonstrates that the CS-emotion link primarily cited in previous self-report and clinical case studies of emotional experiences can extend to actual naturalistic language and facial emotion behaviors. Future studies with multi-modal measures of emotion (e.g., vocal acoustics, skin conductance response) could demonstrate more robust findings. Fourth, due to the parentdirected nature of the study task, we were unable to observe child CS. While it is unlikely that parents' CS was triggered by child's CS given its infrequent occurrence, the child CS-emotion link and its relation to parent behavior remain to be tested. Finally, the present study was conducted to establish naturalistic CS-emotion associations using an ecologically valid observational method. A future experiment with randomized design (e.g., assignment into no CS, single CS, and multiple CS conditions) could further clarify the effects of CS on emotion.

Nonetheless, the present study represents a first step to illuminate the complex links between language and emotion among bilingual speakers in a naturalistic context. We expect our findings on negative emotion predicting CS to generalize to other language combinations, since the cognitive control perspective applies to languages that vary in the order of acquisition (e.g., L1 vs. L2), rather than to specific languages (e.g., Chinese vs. English). Further, the puzzle box 
task that we used in the present study is an ecologically valid measure for eliciting facial emotion, as it mimics real-life parent-child interaction in which the parent must verbally guide their child to complete a difficult task that requires cooperation. This context generalizes to many daily life situations, from explaining to a young child how to ride a bicycle to guiding a student through a challenging homework assignment. This context may further be generalized to other dyads, such as clinicians teaching patients strategies to cope during emotional situations. Based on our findings, CS could be one indicator of a bilingual patient's emotional intensity. It may be valuable for clinicians conducting psychotherapy or text-based mental health apps to monitor language switches as a proxy of their patient's changes in emotion, especially if information from facial behaviors are lacking. Both early (Marcos \& Alpert, 1976; Rozensky \& Gomez, 1983) and recent work (Altarriba \& Morier, 2004) suggests that the strategic use of CS in psychotherapy can have benefits. For example, clinicians may prompt the use of more emotional L1 to increase patient's emotional awareness and expression, and less emotional L2 for cognitive restructuring. Indeed, Spanish/English clinician CS has been found to increase alliance, promote changes in psychotherapeutic strategies, and facilitate patient's disclosure and expression of emotion (Santiago-Rivera et al., 2009).

In conclusion, this is one of the first studies to empirically test the temporally dynamic associations between CS and facial emotion behavior. Our findings provide empirical support for a link between CS and facial emotion behavior, and provide insights into why bilingual speakers code-switch during emotional experiences. To further clarify the relation between CS and emotion, future studies are needed to test the role of arousal (e.g., anger vs. sadness) on CS, effects of CS types (e.g., insertion vs. alternation vs. dense CS) on facial emotion, and predictors of individual differences (e.g., cognitive control) in these links. Given the increasing number of 
cross-linguistic interactions in our global society, and the importance of language as the medium of emotional communication, further empirical research on CS and emotion is warranted.

\section{References}

Abutalebi, J., \& Green, D. (2007). Bilingual language production: The neurocognition of language representation and control. Journal of Neurolinguistics, 20, 242-275. https://doi.org/10.1016/j.jneuroling.2006.10.003

Altarriba, J. \& Morier, R. (2004). Bilingualism: Language, emotion, and mental health. In T. K. Bhatia \& W. C. Ritchie (Eds.), The Handbook of Bilingualism (pp. 250-280). Malden: Blackwell Publishing.

Aragno, A., \& Schlachet, P. (1996). Accessibility of early experiences through the language of origin: A theoretical integration. Psychoanalytic Psychology, 13, 23-34. http://dx.doi.org/10.1037/h0079636

Bates, D., Maechler, M., Bolker, B., \& Walker, S. (2014). lme4: Linear mixed-effects models using Eigen and S4. R Package Version, 1, 1-23.

Bolger, N., Davis, A., \& Rafaeli, E. (2003). Diary methods: Capturing life as it is lived. Annual Review of Psychology, 54, 579-616. http://dx.doi.org/10.1146/annurev.psych.54.101601.145030

Bolger, N. \& Laurenceau, J. P. (2013). Methodology in the social sciences. Intensive longitudinal methods: An introduction to diary and experience sampling research. New York: Guilford Press.

Buriel, R., Love, J., \& De Ment, T. (2006). The relation of language brokering to depression and 
parent-child bonding among Latino adolescents. In M. H. Bornstein \& L. R. Cote (Eds.), Acculturation and parent-child relationships: Measurement and development (pp.249270). Mahwah, NJ: Erlbaum.

Caldwell-Harris, C. (2014). Emotionality differences between a native and foreign language: theoretical implications. Frontiers in Psychology 5 (1055), 1-4. http://dx.doi.org/10.3389/fpsyg.2014.01055

Camras, L., Chen, Y., Bakeman, R., Norris, K., \& Cain, T. R. (2006). Culture, ethnicity, and children's facial expressions: A study of European Americans, Mainland Chinese, Chinese American, and Adopted Chinese girls. Emotion, 6, 103-114.

Chao, R. (1995). Chinese and European American cultural models of the self reflected in mother's childrearing beliefs. Ethos, 23, 328-354.

Chen, X., \& Lee, B. (1996). The Cultural and Social Acculturation Scale. Ontario, Canada: University of Western Ontario.

Chen, S., Kennedy, M., \& Zhou, Q. (2012). Parent's expression and discussion of emotion in the multilingual family: Does language matter? Perspectives on Psychological Science, 7, 365. http://dx.doi.org/10.1177/1745691612447307

Chen, S., Hua, M. Zhou, Q., Tao, A., Lee, E., Ly, J., \& Main, A. (2014). Cultural orientations and child adjustment in Chinese American immigrant families. Developmental Psychology, 50, 189-201.

Chen, S., Zhou, Q., Main, A., \& Lee, E. H. (2015). Chinese American immigrant parent's emotional expression in the family: Relations with parents' cultural orientations and children's emotion-related regulation. Cultural Diversity and Ethnic Minority Psychology, 21, 619-629. http://dx.doi.org/10.1037/cdp0000013 
Clark, H. \& Fox Tree, J. (2002). Using $u h$ and $u m$ in spontaneous speaking. Cognition, 84, 73111.

Cohen, A., Dellarco, D., Breiner, K., Helion, C., Heller, A., Rahdar, A., Pedersen, G., Chein, J., Dyke, J., Galvan, A., \& Casey, B. (2016). The impact of emotional states on cognitive control circuitry and function. Journal of Cognitive Neuroscience, 28, 446-459.

Costa, A., Miozzo, M. \& Caramazza, A. (1999). Lexical selection in bilinguals: Do words in the bilingual's two lexicons compete for selection? Journal of Memory and Language, 41, 365-397. http://dx.doi.org/10.1006/jmla.1999.2651

De Leersnyder, J. \& Mesquita, B. (2017). Emotional frame switching in biculturals: How salient cultural concerns shape emotion. Under Review.

Dewaele, J. (2008). The emotional weight of I love you in multilinguals' languages. Journal of Pragmatics, 40, 1753-1780. https://doi.org/10.1016/j.pragma.2008.03.002

Dewaele, J. (2010). Emotions in Multiple Languages. Basingstoke, UK: Palgrave-Macmillan.

Dewaele, J. (2015). From obscure echo to language of the heart: Multilinguals' language choices for (emotional) inner speech. Journal of Pragmatics, 87, 1-17.

Dewaele, J., \& Nakano, S. (2012). Multilinguals' perceptions of feeling different when switching languages. Journal of Multilingual and Multicultural Development, 34, 107-120. https://doi.org/10.1080/01434632.2012.712133

Dewaele, J., \& Pavlenko, A. (2001-2003). Web questionnaire bilingualism and emotions. London, UK: University of London.

Eisenberg, N., Thompson, E., Gershoff, R., Fabes, S., Shepard, S., Cumberland, A., Losoya, S., 
Guthrie, I., \& Murphy, B. (2001). Mother's emotional expressivity and children's behavior problems and social competence: Mediation through children's regulation. Developmental Psychology, 37, 475-490. http://dx.doi.org/10.1037//0012-1649.37.4.475

Ervin-Tripp, S. (1964). An analysis of the interaction of language, topic, and listener. American Anthropologist, 66, 86-102. https://doi.org/10.1525/aa.1964.66.suppl_3.02a00050

Fabbro, F., Skrap, M., \& Aglioti, S. (2000). Pathological switching between languages after front lesions in a bilingual patient. Journal of Neurology, Neurosurgery, \& Psychiatry, 68, 650-652. http://dx.doi.org/10.1136/jnnp.68.5.650

Fernandez-Dols, J. M. \& Russell, J. (2017). The Science of Facial Expression. New York: Oxford University Press.

Fillmore, L. W. (1991). When leaning a second language means losing the first. Early Childhood Research Quarterly, 6, 323-346.

Foster, R. (1996). The bilingual self: Duet in two voices. Psychoanalytic Dialogues, 6, 99-121.

Gafaranga, J. (2009). The conversation analytic model of CS. In B.E. Bullock \& A.

J. Toribio (Eds.), The Cambridge Handbook of Linguistic CS, (pp. 114-127). New York: Cambridge University Press.

Gardner-Chloros, P. (2009). CS. London, UK: Cambridge University Press.

Garrett-Peters, P., \& Fox, N. (2007). Cross-cultural differences in children's emotional reactions to a disappointing situation. International Journal of Behavioral Development, 31, 161169. http://dx.doi.org/10.1177/0165025407074627

Genesee, F., Nicoladis, E., \& Paradis, J. (1995). Language differentiation in early bilingual development. Journal of Child Language, 22, 611-631.

Green, D. W., \& Abutalebi, J. (2013). Language control in bilinguals: The adaptive control hypothesis. Journal of Cognitive Psychology, 25, 515-530. 
Green, D. W., \& Wei, L. (2014). A control process model of CS. Language, Cognition and Neuroscience, 29(4), 499-511.

Gumperz, J. (1982). Discourse strategies. Cambridge University Press, Cambridge.

Harris, C., Gleason, J., Harris, Ayçiçegi, A. (2006). When is a first language more emotional? Psychophysiological evidence from bilingual speakers. In Aneta Pavlenko (Ed). Bilingual Minds: Emotional Experience, Expression, and Representation, (pp. 257-283). Clevedon, UK: Multilingual Matters.

Javier, R. (1989). Linguistic consideration in the treatment of bilinguals. Journal of Psychoanalytic Psychology, 6, 80-96. http://dx.doi.org/10.1037/0736-9735.6.1.87

Keysar, B., Hayakawa, S., \& An, S. (2012). The foreign language effect: Thinking in a foreign tongue reduces decision biases. Psychological Science, 23, 661-668.

Lausberg, H. \& Sloetjes, H. (2009). Coding gestural behavior with the NEUROGES-ELAN system. Behavior Research Methods, r1, 841-849. http://dx.doi.org/10.3758/BRM.41.3.841

Levenson, R. W. (1999). The intrapersonal functions of emotion. Cognition and Emotion, 13, 481-504. https://doi.org/10.1080/026999399379159

Lindsey E., Creemens, P., Colwell, M., \& Caldera, Y. (2009). The structure of parent-child dyadic synchrony in toddlerhood and children's communication competence and selfcontrol. Social Development, 18, 375-196. https://doi.org/10.1111/j.14679507.2008.00489.x

Lindquist, K. A. \& Barret, L. F. (2008). Constructing emotion: the experience of fear as a conceptual act. Psychological Science, 19, 898-903. http://dx.doi.org/10.1111/j.14679280.2008.02174.x 
Lindquist, K. A., Gendron, M., \& Satpute, A. B. (2018). Language and Emotion: Putting Words into Feelings and Feelings into Words. In L.F. Barrett, M. Lewis \& J. M. Haviland-Jones (Eds.), Handbook of Emotions. New York, NY: Guilford.

Marcos, L., \& Alpert, M. (1976). Strategies and risks in psychotherapy with bilingual patients: The phenomenon of language independence. American Journal of Psychiatry, 133, 12751278. http://dx.doi.org/10.1176/ajp.133.11.1275

Matsumoto, D. (1993). Ethnic differences in affect intensity, emotion judgments, display rule attitudes, and self-reported emotional expression in an American sample. Motivation and Emotion, 17, 107-123.

Mauss, I. B., Levenson, R. W., McCarter, L., Wilhelm F., Gross, J. J. (2005). The tie that binds? Coherence among emotion experience, behavior, and physiology. Emotion 5(2), 175-190. $10.1037 / 1528-3542.5 .2 .175$

Meisel, J. (1994). CS in young bilingual children: The acquisition of grammatical constraints. Studies in Second Language Acquisition, 16, 413-439. https://doi.org/10.1017/S0272263100013449

Mesquita, B., Boiger, M., \& De Leersnyder, J. (2017). Doing emotions: The role of culture in everyday emotions. European Review of Social Psychology, 28, 95-113. https://doi.org/10.1080/10463283.2017.1329107

Mohavedi, S. (1996). Metalinguistic analysis of therapeutic discourse: Flight into a second language when the analyst and analysand are multilingual. Journal of the American Psychoanalytic Association, 44(3), 837-862.

Myers-Scotton, C. (1993). Common and uncommon ground: Social and structural factors in 
codeswitching. Language in Society, 22, 475-503.

http://dx.doi.org/10.1017/S0047404500017449

Mueller, S. (2011). The influence of emotion on cognitive control: Relevance for development and adolescent psychopathology. Frontiers in Psychology, 2, 1-21. http://dx.doi.org/ 10.3389/fpsyg.2011.00327

Muthén, L. \& Muthén, B. (2002). How to use a Monte Carlo study to decide on sample size and determine power. Structural Equation Modeling, 4, 599-620. https://doi.org/10.1207/S15328007SEM0904_8

Muysken, P. (2000). Bilingual Speech: A Typology of Code Mixing. Cambridge, UK: Cambridge University Press.

National Academies of Sciences, Engineering, and Medicine. (2017). Promoting the Educational Success of Children and Youth Learning English: Promising Futures. Washington, DC: The National Academies Press.

Ng, S. \& He, A. (2004). CS in trigenerational family conversations among Chinese immigrants in New Zealand. Journal of Language and Social Psychology, 23, 28-48.

Panayiotou, A. (2004). Switching codes, switching code: Bilinguals' emotional response in English and Greek. Journal of Multilingual and Multicultural Development, 25, 124-139. https://doi.org/10.1080/01434630408666525

Pavlenko, A. (2004). 'Stop doing that, Ia komu skazala!: Language choice and emotions in parent-child communication. Journal of Multilingual and Multicultural Development, 25, 179-203. http://dx.doi.org/10.1080/01434630408666528

Pavlenko, A. (2005). Emotions and multilingualism. Cambridge, UK: Cambridge University Press. 
Pavlenko, A., (2012). Affective processing in bilingual speakers: Disembodied cognition? International Journal of Psychology, 47, 405-428. http:dx.doi.org/10.1080/00207594.2012.743665

Pinheiro, J., Bates, D., DebRoy, S., Sarkar, D., Heisterkamp, S., Van Willigen, B., \& Maintainer, R. (2017). Package 'nlme'. Linear and Nonlinear Mixed Effects Models, 3-1.

Poplack, S. (1980). Sometimes I'll start a sentence in Spanish Y TERMINO EN ESPANOL: toward a typology of CS. Linguistics, 18, 581-618.

R Core Team. (2013). R: A language and environment for statistical computing. R Foundation for Statistical Computing, Vienna, Austria. Retrieved from http://www.R-project.org/

Roosa, M., Liu, F, Torres, M., Gonzales, N., Knight, G., \& Saenz, D. (2008). Sampling and recruitment in studies of cultural influences on adjustment: A case study with Mexican Americans. Journal of Family Psychology, 22, 293-302. http://dx.doi.org/10.1037/08933200.22 .2 .293

Ribot, K. \& Hoff, E. (2014). ¿Cómo estas?” “I’m good.” Conversational code-switching is related to profiles of expressive and receptive proficiency in Spanish-English bilingual toddlers. International Journal of Behavioral Development, 38(4), 333-341.

Ross, M., Xun, W. Q., Wilson, A. (2002). Language and the bicultural self. Personality and Social Psychology Bulletin, 28, 1040-1050. https://doi.org/10.1177/01461672022811003

Rozensky, R., \& Gomez, M. (1983). Language switching in psychotherapy with bilinguals: Two problems, two models, and case examples. Psychotherapy: Theory, Research \& Practice, 20, 152-160. http://dx.doi.org/10.1037/h0088486

Santiago-Rivera, A., \& Altarriba, J. (2002). The role of language in therapy with the SpanishEnglish bilingual client. Professional Psychology: Research and Practice, 33, 30-38. 
Santiago-Rivera, A., Altarriba, J., Poll, N., Gonzalez-Miller, N., Cragun, C. (2009). Therapists' views on working with bilingual Spanish-English clients: A qualitative investigation. Professional Psychology: Research and Practice, 40, 436-443.

Snijders, T., \& Bosker, R. (1999). Multilevel analysis: An introduction to basic and applied multilevel analysis. London, UK: Sage.

Soto, J. A., Levenson R. W., \& Ebling, R. (2005). Cultures of moderation and expression: Emotional experience, behavior, and physiology in Chinese Americans and Mexican Americans. Emotion, 5, 154-165. http://dx.doi.org/10.1037/1528-3542.5.2.154

Thoma, D., \& Baum, A. (2018) Reduced language processing automaticity induces weaker emotions in bilinguals regardless of learning context. Emotion. Advance online publication. http://dx.doi.org/10.1037/emo0000502

Tottenham, N., Hare, T. A., \& Casey, B. J. (2011). Behavioral assessment of emotion discrimination, emotion regulation, and cognitive control in childhood, adolescence, and adulthood. Frontiers in Psychology, 2(39), 1-7.

Tsai, J. (2007). Ideal affect. Perspectives on Psychological Science, 2, 242-259.

Walden, T. A., Frankel, C. B., Buhr, A. P., Johnson, K. N., Conture, E. G., \& Karrass, J. M. Dual diathesis-stressor model of emotional and linguistic contributions to developmental stuttering. Journal of Abnormal Child Psychology, 40, 633-644. http://dx.doi.org/10.1007/s10802-011-9581-8

Wang, Q., Shao, Y., \& Li, Y. (2010). “My way or mom's way?” The bilingual and bicultural self in Hong Kong Chinese children and adolescents. Child Development, 81, 555-567.

Wierzbicka, A. (2005). Universal human concepts as a tool for exploring bilingual lives. International Journal of Bilingualism, 9, 7-26. 


\section{Table 1}

Results of Multilevel Model Testing if Parent's Positive or Negative Emotion Predicts CS

\begin{tabular}{|c|c|c|c|}
\hline Parent's CS Frequency & Estimate (SE) & $p$ & $95 \% \mathrm{CI}$ \\
\hline \multicolumn{4}{|c|}{ Positive Facial Emotion Predicting CS } \\
\hline \multicolumn{4}{|c|}{ Fixed Effects } \\
\hline Intercept $\boldsymbol{\gamma}_{\mathbf{0 0}}$ & $1.24(.12)$ & $<.001$ & {$[1.01,1.47]$} \\
\hline $\mathrm{CS}, \mathrm{t}-1 \gamma_{\mathbf{1 0}}$ & $.17(.03)$ & $<.001$ & {$[.11, .23]$} \\
\hline State PE, t $\gamma_{20}$ & $-.10(.06)$ & .08 & {$[-.22, .01]$} \\
\hline State PE, $\mathrm{t}-1 \boldsymbol{\gamma}_{\mathbf{3 0}}$ & $-.08(.06)$ & .17 & {$[-.20, .03]$} \\
\hline Mean PE $\boldsymbol{\gamma}_{\mathbf{0 1}}$ & $.01(.25)$ & .98 & {$[-.50, .51]$} \\
\hline \multicolumn{4}{|l|}{ Random Effects } \\
\hline SD of Intercept $\boldsymbol{\sigma}_{\boldsymbol{u} \mathbf{0}}$ & .58 & & {$[.42, .79]$} \\
\hline SD of CS, t-1 $\sigma_{u 1}$ & .07 & & {$[.01, .30]$} \\
\hline Correlation $r_{u 0 u 1}$ & -.90 & & {$[-.99, .98]$} \\
\hline Residual SD $\boldsymbol{\sigma}_{\boldsymbol{e}}$ & 1.42 & & {$[1.36,1.47]$} \\
\hline$-2 \mathrm{LL}$ & -2497.45 & & \\
\hline \multicolumn{4}{|c|}{ Negative Facial Emotion Predicting CS } \\
\hline \multicolumn{4}{|l|}{ Fixed Effects } \\
\hline Intercept $\boldsymbol{\gamma}_{\mathbf{0 0}}$ & $1.23(.11)$ & $<.001$ & {$[1.02,1.45]$} \\
\hline $\mathrm{CS}, \mathrm{t}-1 \gamma_{\mathbf{1 0}}$ & $.17(.03)$ & $<.001$ & {$[.11, .23]$} \\
\hline State NE, t $\gamma_{20}$ & $.04(.09)$ & .64 & {$[-.13, .21]$} \\
\hline State NE, $\mathrm{t}-1 \gamma_{\mathbf{3 0}}$ & $.20(.09)$ & .02 & {$[.03, .37]$} \\
\hline Mean NE $\boldsymbol{\gamma}_{01}$ & $.58(.65)$ & .38 & {$[-.76,1.91]$} \\
\hline \multicolumn{4}{|l|}{ Random Effects } \\
\hline SD of Intercept $\boldsymbol{\sigma}_{\boldsymbol{u} \mathbf{0}}$ & .55 & & {$[.40, .76]$} \\
\hline $\mathrm{SD}$ of CS, $\mathrm{t}-1 \boldsymbol{\sigma}_{\boldsymbol{u} \mathbf{1}}$ & .06 & & {$[.01, .37]$} \\
\hline Covariance $r_{u 0 u 1}$ & -.77 & & {$[-.99, .85]$} \\
\hline $\begin{array}{l}\text { Residual SD } \boldsymbol{\sigma}_{\boldsymbol{e}} \\
-2 \mathrm{LL}\end{array}$ & $\begin{array}{c}1.42 \\
-2495.58\end{array}$ & & {$[1.36,1.47]$} \\
\hline
\end{tabular}

Notes. Unstandardized estimates. $\mathrm{SE}=$ standard error. $95 \% \mathrm{CI}=95 \%$ confidence interval. $\sigma=$ standard deviation of random effects. $\mathrm{CS}=\mathrm{CS} . \mathrm{PE}=$ positive facial emotion behavior. $\mathrm{NE}=$ negative facial emotion behavior. 
Table 2

Results of Multilevel Model Testing if Parent's CS Predicts Positive or Negative Emotion

\begin{tabular}{|c|c|c|c|c|}
\hline Parent's Facial Emotion Behavior & Estimate (SE) & $p$ & $95 \% \mathrm{CI}$ & Exp. (Estimate) \\
\hline \multicolumn{5}{|c|}{ CS Predicting Positive Facial Emotion } \\
\hline \multicolumn{5}{|c|}{ Fixed Effects } \\
\hline Intercept $\boldsymbol{\gamma}_{\mathbf{4 0}}$ & $-1.66(.19)$ & $<.001$ & {$[-2.06,-1.29]$} & .19 \\
\hline $\mathrm{PE}, \mathrm{t}-1 \gamma_{\mathbf{5 0}}$ & $.61(.09)$ & $<.001$ & {$[.42, .78]$} & 1.84 \\
\hline State CS, t $\gamma_{60}$ & $-.06(.03)$ & .11 & {$[-.12, .01]$} & .94 \\
\hline State CS, t-1 $\gamma_{\mathbf{7 0}}$ & $-.04(.03)$ & .19 & {$[-.11, .02]$} & .96 \\
\hline Mean CS $\gamma_{41}$ & $.17(.23)$ & .46 & {$[-.38, .60]$} & 1.19 \\
\hline \multicolumn{5}{|l|}{ Random Effects } \\
\hline SD of Intercept $\boldsymbol{\sigma}_{\boldsymbol{u} \boldsymbol{4}}$ & .98 & & {$[.71,1.39]$} & 2.66 \\
\hline SD of PE, t-1 $\boldsymbol{\sigma}_{\boldsymbol{u} \mathbf{5}}$ & .26 & & {$[.13, .42]$} & 1.30 \\
\hline \multirow{2}{*}{$\begin{array}{l}\text { Correlation } \boldsymbol{r}_{\boldsymbol{u 4 u \mathbf { 5 }}} \\
\text {-2LL }\end{array}$} & -.96 & & {$[-1.00,-.37]$} & .38 \\
\hline & -974.20 & & & \\
\hline \multicolumn{5}{|c|}{ CS Predicting Negative Facial Emotion } \\
\hline \multicolumn{5}{|l|}{ Fixed Effects } \\
\hline Intercept $\boldsymbol{\gamma}_{\mathbf{4 0}}$ & $-2.03(.13)$ & $<.001$ & {$[-2.31,-1.79]$} & .13 \\
\hline $\mathrm{NE}, \mathrm{t}-1 \gamma_{\mathbf{5 0}}$ & $.85(.12)$ & $<.001$ & {$[.61,1.11]$} & 2.34 \\
\hline State CS, t $\gamma_{60}$ & $.02(.04)$ & .64 & {$[-.06, .09]$} & 1.02 \\
\hline State CS, t- $1 \gamma_{\mathbf{7 0}}$ & $.02(.04)$ & .58 & {$[-.06, .10]$} & 1.02 \\
\hline Mean CS $\gamma_{41}$ & $.24(.16)$ & .13 & {$[-.06, .60]$} & 1.27 \\
\hline \multicolumn{5}{|l|}{ Random Effects } \\
\hline SD of Intercept $\boldsymbol{\sigma}_{\boldsymbol{u} \boldsymbol{4}}$ & .54 & & {$[.33, .82]$} & 1.72 \\
\hline SD of NE, t- $1 \boldsymbol{\sigma}_{\boldsymbol{u} \mathbf{5}}$ & .20 & & {$[.00, .45]$} & 1.22 \\
\hline \multirow{2}{*}{$\begin{array}{l}\text { Covariance } \boldsymbol{r}_{\boldsymbol{u 4 u 5}} \\
\text {-2LL }\end{array}$} & -1.00 & & {$[-1.00,1.00]$} & .37 \\
\hline & -693.10 & & & \\
\hline
\end{tabular}

Notes. Unstandardized estimates. $\mathrm{SE}=$ standard error. 95\% CI $=95 \%$ confidence interval. Exp. $($ estimate $)=$ exponentiated parameter values for ease of interpretation. $\sigma=$ standard deviation of random effects. $\mathrm{CS}=\mathrm{CS} . \mathrm{PE}=$ positive facial emotion behavior. $\mathrm{NE}=$ negative facial emotion behavior. 